\title{
Overview of $\Upsilon$ production studies performed with the STAR experiment
}

\section{Leszek Kosarzewski* for the STAR collaboration}

Faculty of Nuclear Sciences and Physical Engineering, Czech Technical University in Prague, Břehová 78/7, 11519 Staré Město, Prague, Czech Republic

E-mail: kosarles@fjfi.cvut.cz

The $\Upsilon$ states are a clean probe of the properties of quark-gluon plasma, which can be created in heavy-ion collisions. Each of the $\Upsilon$ states dissociates at a different temperature in the plasma due to Debye-like screening of the quark-antiquark potential by the surrounding color charges. In order to understand the Cold Nuclear Matter effects, the $\Upsilon$ production has to be studied in small collision systems such as $\mathrm{p}+\mathrm{Au}$ or $\mathrm{d}+\mathrm{Au}$ collisions. Measurements of $\Upsilon$ production cross section in $\mathrm{p}+\mathrm{p}$ collisions allow one to study the production mechanism, while its dependence on charged particle multiplicity provides information on the interplay of hard and soft QCD processes.

In this contribution, an overview of the measurements of the production of $\Upsilon$ states done by the STAR experiment is presented. This includes the rapidity spectra and transverse momentum spectra of different states in $\mathrm{p}+\mathrm{p}$ collisions at $\sqrt{s}=500 \mathrm{GeV}$. Nuclear modification factors measured in $\mathrm{p}+\mathrm{Au}, \mathrm{d}+\mathrm{Au}$ and $\mathrm{Au}+\mathrm{Au}$ collisions at $\sqrt{s_{N N}}=200 \mathrm{GeV}$ are also shown.

The $\Upsilon(1 S)$ spectra in $\mathrm{p}+\mathrm{p}$ collisions are reasonably described by the Color Evaporation Model calculation, while overestimated by calculations based on Non-Relativistic QCD formalism coupled with the Color Glass Condensate effective theory. In Au+Au collisions, the $\Upsilon(1 S)$ is similarly suppressed to what observed at the LHC, while $\Upsilon(2 S+3 S)$ are more suppressed than $\Upsilon(1 S)$ in central collisions. This is an indication of sequential suppression of $\Upsilon$ states at $\sqrt{s_{N N}}=200 \mathrm{GeV}$. The model calculations are consistent with the data.

40th International Conference on High Energy physics - ICHEP2020

July 28 - August 6, 2020

Prague, Czech Republic (virtual meeting)

\footnotetext{
${ }^{*}$ Speaker
} 


\section{Introduction}

Upsilon $(\Upsilon)$ states are clean probes of the quark-gluon plasma (QGP), a state of matter that is believed to have existed in the early Universe and can be recreated in heavy-ion collisions. Within a static dissociation picture, their production in high energy heavy-ion collisions is expected to be reduced with respect to $p+p$ collisions due to a Debye-like screening of the quark-antiquark potential by the color charges in the high temperature QGP medium [1]. Each of the $\Upsilon$ states has a different binding energy, so dissociates at a different temperature. The yield of each state contains a contribution from the decay of excited states, so called the feed-down contribution, for example $\Upsilon(n S) \rightarrow \Upsilon(1 S) \pi^{+} \pi^{-}, \chi_{\mathrm{bJ}}(n S) \rightarrow \Upsilon(1 S) \gamma$ and similar.

The production of $\Upsilon$ states in vacuum is not yet fully understood. A common assumption is that the production can be described as a two-step process in a factorized scheme, with a $b \bar{b}$ pair produced in a hard QCD process followed by the subsequent evolution into a bound state, which is a soft process. The latter is not yet well understood, but it takes place through intermediate color singlet (CS) or color octet (CO) channels. Measurements of $\Upsilon$ production in $\mathrm{p}+\mathrm{p}$ collisions allow one to verify the production models and serve as a baseline for studies in heavy-ion collisions.

There are also various Cold Nuclear Matter (CNM) effects, which affect the observed yield of $\Upsilon$ mesons. First of all, the $b \bar{b}$ pair production cross section depends on the gluon distribution in a nucleon, which is modified for bound nucleons compared to free nucleons (shadowing, antishadowing). Furthermore, the $\Upsilon$ states may be broken up during interactions with co-moving hadrons, but this is estimated to be a small effect for $\Upsilon(1 S)$ [2]. Breaking-up due to interactions with nucleons in a nucleus is also possible [3]. These effects can be studied in $\mathrm{p}+\mathrm{A}$ or $\mathrm{d}+\mathrm{A}$ collisions.

Finally, all the above effects are present in A+A collisions with a few additions - regeneration, medium induced energy loss and parton fragmentation [3]. Regeneration is however expected to be negligible for $\Upsilon$ at RHIC energies because of the very low $b \bar{b}$ pair production cross section. In order to fully understand the interplay between these effects, a detailed and differential study has to be performed in different collision systems and at different collision energies.

\section{Upsilon measurements with the STAR experiment}

In the STAR experiment, the $\Upsilon$ production is studied in the dielectron $\left(e^{+} e^{-}\right)$and dimuon $\left(\mu^{+} \mu^{-}\right)$ decay channels. A signal candidate is reconstructed from a pair of tracks in the Time Projection Chamber (TPC), which is the main tracking detector, responsible for momentum measurement and charged particle identification. Further identification of electrons is done using the Barrel Electromagnetic Calorimeter (BEMC). At least one track has to be matched to a high energy hit in the BEMC, that fired a trigger. The electrons are measured within the pseudorapidity range of $|\eta|<1$. Muon tracks are identified using the Muon Telescope Detector (MTD), which is located outside of the STAR magnet acting as an absorber for hadrons. A dimuon trigger was implemented by requiring at least 2 hits in the MTD. This system has a coverage of approximately $45 \%$ acceptance in azimuthal angle $\phi$ for $|\eta|<0.5$. 

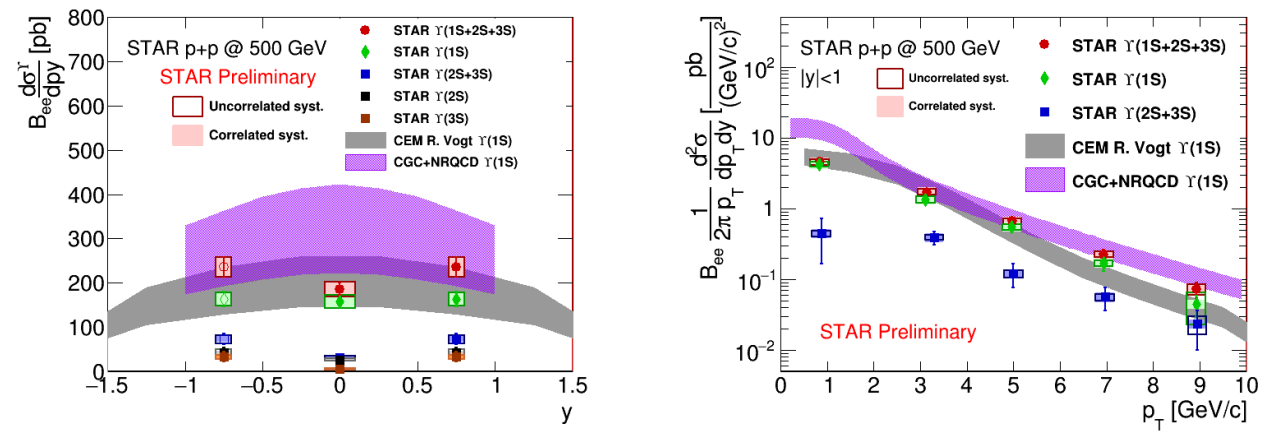

Figure 1: Production cross section for $\Upsilon(n S)$ states vs. $y$ (left) and $p_{\mathrm{T}}$ (right). Data are compared to CEM [4] and CGC+NRQCD [5, 6] model calculations for inclusive and direct $\Upsilon(1 S)$ respectively.

\section{Upsilon production in $\mathbf{p}+\mathbf{p}$ collisions}

The production of the $r$ states was measured by STAR in the dielectron channel at both $\sqrt{s}=200 \mathrm{GeV}$ and $\sqrt{s}=500 \mathrm{GeV}$. Figure 1 (left) shows the rapidity $(y)$ dependence of the production cross section for the $\Upsilon(n S)$ states compared to the Color Evaporation Model (CEM) [4] calculations for inclusive $\Upsilon(1 S)$ as well as Non-Relativistic QCD formalism coupled to Color Glass Condensate effective theory (CGC+NRQCD) $[5,6]$ for direct $\Upsilon(1 S)$. Similarly, the right panel of Fig. 1 shows the transverse momentum $\left(p_{\mathrm{T}}\right)$ dependence of the $\Upsilon(n S)$ production cross section, which is also compared to the models. The data are reasonably well described by the CEM model, while overestimated by CGC+NRQCD, especially for $p_{\mathrm{T}}<2 \mathrm{GeV} / \mathrm{c}$.
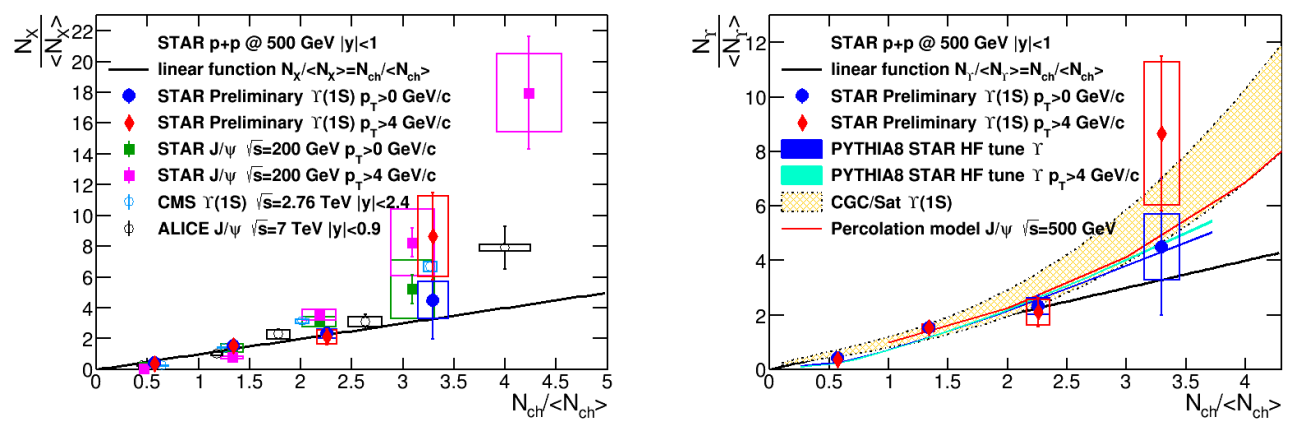

Figure 2: Self-normalized yield $\Upsilon(1 S) /\langle\Upsilon(1 S)\rangle$ vs. self-normalized multiplicity $N_{\mathrm{ch}} /\left\langle N_{\mathrm{ch}}\right\rangle$. The results are compared to other measurements [7-9] (left) and model calculations (right) [10, 11].

Measurements of charged particle multiplicity $\left(N_{\mathrm{ch}}\right)$ dependence of $\gamma$ production allow one to study the interplay between hard and soft processes in $\mathrm{p}+\mathrm{p}$ collisions. A self-normalized $\gamma$ yield $\Upsilon(1 S) /\langle\Upsilon(1 S)\rangle$ vs. self-normalized multiplicity $N_{\mathrm{ch}} /\left\langle N_{\mathrm{ch}}\right\rangle$ (both measured in $|\eta|<1$ ) was measured by STAR at $\sqrt{s}=500 \mathrm{GeV}$. The results are shown in Fig. 2 and compared to a similar measurement by CMS [7] and $J / \psi$ measured by STAR [8] and ALICE [9]. In Fig. 2, the same data are compared to a String Percolation model [10], a CGC/Saturation-based approach [11] and PYTHIA8 calculations. All models qualitatively reproduce the trend observed both in $J / \psi$ and $\Upsilon$ data at RHIC and LHC. 


\section{Upsilon production in $\mathrm{p}+\mathrm{Au}$ and $\mathrm{d}+\mathrm{Au}$ collisions}

In order to quantify the CNM effects, the nuclear modification factor $\left(R_{\mathrm{pA}}\right)$ is used.

$$
R_{\mathrm{pA}}=\frac{1}{\left\langle N_{\text {coll }}\right\rangle} \frac{\mathrm{d}^{2} N_{\mathrm{p}+\mathrm{A}} / \mathrm{d} p_{\mathrm{T}} \mathrm{d} y}{\mathrm{~d}^{2} N_{\mathrm{p}+\mathrm{p}} / \mathrm{d} p_{\mathrm{T}} \mathrm{d} y}
$$

It is the ratio of the yield of $\Upsilon$ measured in $\mathrm{p}+\mathrm{A}\left(N_{\mathrm{p}+\mathrm{A}}\right)$ collisions to the one measured in $\mathrm{p}+\mathrm{p}\left(N_{\mathrm{p}+\mathrm{p}}\right)$ collisions, scaled by the number of binary nucleon-nucleon collisions $\left(\left\langle N_{\text {coll }}\right\rangle\right)$.

STAR has studied $\Upsilon$ production in $\mathrm{p}+\mathrm{Au}$ and $\mathrm{d}+\mathrm{Au}$ collisions at $\sqrt{s_{\mathrm{NN}}}=200 \mathrm{GeV}$ in order to study the CNM effects. The obtained nuclear modification factor $R_{\mathrm{pA}}\left(R_{\mathrm{dA}}\right)$ [12] vs. rapidity is shown in Fig. 3. The data are compared to the same measurements by PHENIX [13] and to models taking into account the effect of modified nuclear

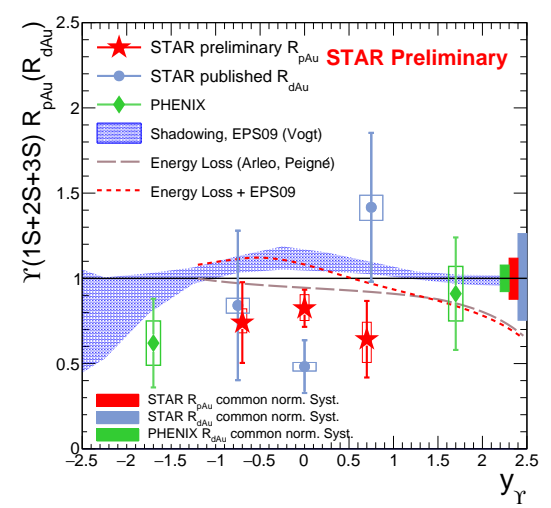

Figure 3: $\quad$ The nuclear modification factor $R_{\mathrm{pA}}\left(R_{\mathrm{dA}}\right)$ of $\Upsilon$ measured by STAR [12] and PHENIX [13] compared to model calculations $[14,15]$. PDFs (nPDFs) [14] only, as well as energy loss in addition to nPDFs or energy loss only [15]. The data show a suppression in $\mathrm{p}+\mathrm{Au}$ collisions indicating that the CNM effects are not negligible.

\section{Upsilon production in Au+Au collisions}

The yields of $\Upsilon$ in Au+Au collisions at $\sqrt{s_{\mathrm{NN}}}=200 \mathrm{GeV}$ were measured in both dielectron and dimuon channels and combined for better precision. The collected data sample was sufficient to calculate $R_{\mathrm{AA}}$ vs. number of participating nucleons $N_{\text {part }}$ separately for $\Upsilon(1 S)$ and $\Upsilon(2 S+3 S)$, which are shown in Fig. 4 (left) and Fig. 4 (right) respectively. The results are compared to measurements by CMS [16]. At RHIC, the $R_{\mathrm{AA}}$ of $\Upsilon(1 S)$ is similar to the one measured at the LHC and the $\Upsilon(2 S+3 S)$ states are more suppressed than $\Upsilon(1 S)$ in central collisions.

The STAR data are also compared to the model calculations, which are shown in Fig. 5. The calculation by Rothkopf [17] does not include regeneration and CNM effects, while both effects are included in Rapp's calculation [18]. All these models include the effect of Debye-like screening.
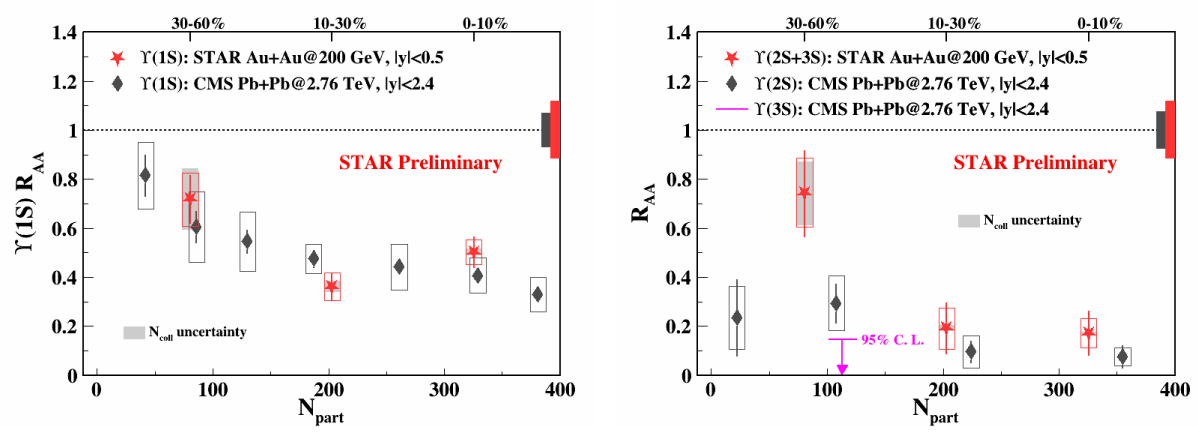

Figure 4: Nuclear modification factor $R_{\mathrm{AA}}$ for $\Upsilon(1 S)$ (left) and $\Upsilon(2 S+3 S)$ (right) vs. $N_{\text {part }}$ compared to the CMS results [16]. 

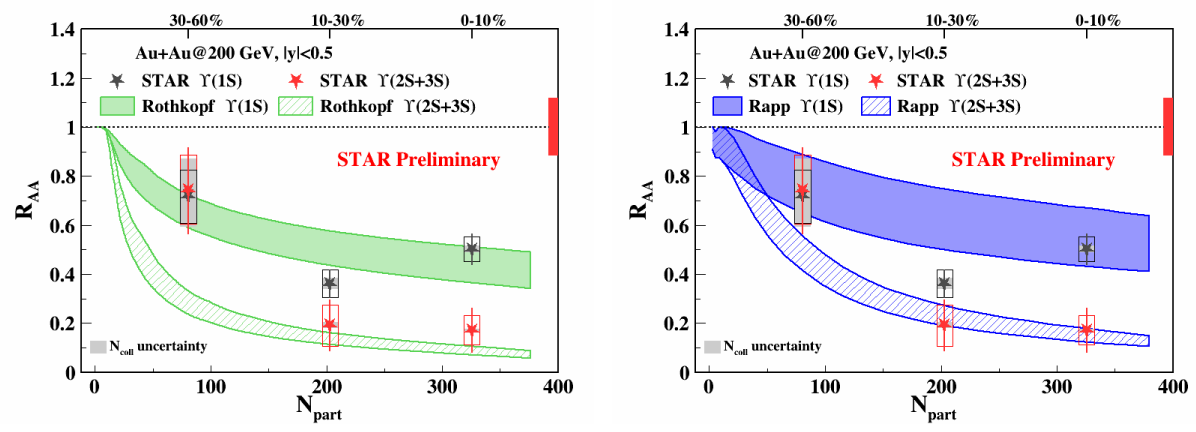

Figure 5: $\quad$ Nuclear modification factor $R_{\mathrm{AA}}$ for $\Upsilon(1 S)$ and $\Upsilon(2 S+3 S)$ vs. $\quad N_{\text {part }}$ compared to model calculations $[17,18]$.

Data can be qualitatively described by model calculations, except for a hint of discrepancy with Rothkopf's model for $\Upsilon(2 S+3 S)$ in $30-60 \%$ centrality class.

\section{Conclusions}

A comprehensive study of the $\Upsilon$ production was performed in STAR in $\mathrm{p}+\mathrm{p}, \mathrm{p}+\mathrm{Au}, \mathrm{d}+\mathrm{Au}$, and $\mathrm{Au}+\mathrm{Au}$ collisions. The measurements of $\gamma$ cross section in $\mathrm{p}+\mathrm{p}$ are reasonably well described by the CEM model calculation, while overestimated by CGC+NRQCD mostly for $p_{\mathrm{T}}<2 \mathrm{GeV} / \mathrm{c}$. A strong increase is observed for $\Upsilon(1 S) /\langle\Upsilon(1 S)\rangle$ vs. $N_{\mathrm{ch}} /\left\langle N_{\mathrm{ch}}\right\rangle$, which follows the trend observed at the LHC and is similar to that of $J / \psi$. The $R_{\mathrm{pA}}$ of $\Upsilon$ shows an indication of suppression, which suggests that the CNM effects could play a role in the suppression observed in $\mathrm{Au}+\mathrm{Au}$ collisions. The $R_{\mathrm{AA}}$ of $\Upsilon(1 S)$ in $\mathrm{Au}+\mathrm{Au}$ collisions at $\sqrt{s_{\mathrm{NN}}}=200 \mathrm{GeV}$ is similar to the one measured by CMS. This similarity suggests that the observed suppression at both energies is mainly due to the suppression of feed-down contribution to $\Upsilon(1 S)$ and CNM effects. On the other hand, the $R_{\mathrm{AA}}$ of $\Upsilon(2 S+3 S)$ is smaller than $\Upsilon(1 S)$ in central $\mathrm{Au}+\mathrm{Au}$ collisions, an indication of sequential suppression of $\Upsilon$ states at RHIC energy. The STAR $R_{\mathrm{AA}}$ data can be qualitatively described by the theoretical models.

\section{Acknowledgements}

This work was also supported from European Regional Development Fund-Project "Center of Advanced Applied Science" No. CZ.02.1.01/0.0/0.0/16-019/0000778 and by the grant LTT18002 of Ministry of Education, Youth and Sports of the Czech Republic.

\section{References}

[1] T. Matsui and H. Satz, J/ $\psi$ suppression by quark-gluon plasma formation, Physics Letters $B$ 178 (1986) 416.

[2] Z. Lin and C. Ko, $\Upsilon$ absorption in hadronic matter, Physics Letters B 503 (2001) 104 [nucl-th/0007027].

[3] A. Andronic, F. Arleo, R. Arnaldi, A. Beraudo, E. Bruna, D. Caffarri et al., Heavy-flavour and quarkonium production in the LHC era: from proton-proton to heavy-ion collisions, The European Physical Journal C 76 (2016) 107 [1506.03981]. 
[4] R. Vogt, Shadowing effects on $J / \psi$ and $\Upsilon$ production at energies available at the CERN Large Hadron Collider, Phys. Rev. C 92 (2015) 034909 [1507. 04418].

[5] H. Han, Y.-Q. Ma, C. Meng, H.-S. Shao, Y.-J. Zhang and K.-T. Chao, $\Upsilon(n S)$ and $\chi_{b}(n P)$ production at hadron colliders in nonrelativistic QCD, Phys. Rev. D 94 (2016) 014028 [1410.8537].

[6] Y.-Q. Ma and R. Venugopalan, Comprehensive description of $J / \psi$ production in proton-proton collisions at collider energies, Phys. Rev. Lett. 113 (2014) 192301 [1408.4075].

[7] Chatrchyan, S. et. al, Event activity dependence of $\Upsilon(n S)$ production in $\sqrt{s}=5.02 \mathrm{TeV} \mathrm{pPb}$ and $\sqrt{s}=2.76 \mathrm{TeV}$ pp collisions, Journal of High Energy Physics 2014 (2014) 103 [1312.6300].

[8] J. Adam et. al, $J / \psi$ production cross section and its dependence on charged-particle multiplicity in $p+p$ collisions at $\sqrt{s}=200 \mathrm{GeV}$, Physics Letters B 786 (2018) 87 [1805.03745].

[9] B. Abelev et. al, $J / \psi$ production as a function of charged particle multiplicity in $p p$ collisions at $\sqrt{s}=7 \mathrm{TeV}$, Physics Letters B 712 (2012) 165 [1202 .2816].

[10] E.G. Ferreiro and C. Pajares, High multiplicity pp events and $J / \psi$ production at LHC, Physical Review C 86 (2012) 034903 [1203 . 5936].

[11] E. Levin, I. Schmidt and M. Siddikov, Multiplicity dependence of quarkonia production in the CGC approach, The European Physical Journal C 80 (2020) 560 [1910 . 13579].

[12] L. Adamczyk et. al, Suppression of $\Upsilon$ production in $d+A u$ and $A u+A u$ collisions at $\sqrt{s_{N N}}=200 \mathrm{GeV}$, Physics Letters B 735 (2014) 127 [1312.3675].

[13] PHENIX Collaboration collaboration, $\Upsilon(1 s+2 s+3 s)$ production in $d+A u$ and $p+p$ collisions at $\sqrt{s_{N N}}=200 \mathrm{GeV}$ and cold-nuclear-matter effects, Phys. Rev. C 87 (2013) 044909 [1211.4017].

[14] R. Vogt, private communication, 2014.

[15] F. Arleo and S. Peigné, Heavy-quarkonium suppression in p-A collisions from parton energy loss in cold QCD matter, Journal of High Energy Physics 2013 (2013) 122 [1212 . 0434].

[16] V. Khachatryan, A. Sirunyan, A. Tumasyan, W. Adam, E. Asilar, T. Bergauer et al., Suppression of $\Upsilon(1 S), \Upsilon(2 S)$, and $\Upsilon(3 S)$ quarkonium states in PbPb collisions at $\sqrt{s_{N N}}=$ $2.76 \mathrm{TeV}$, Physics Letters B 770 (2017) 357 [1611.01510].

[17] B. Krouppa, A. Rothkopf and M. Strickland, Bottomonium suppression using a lattice qcd vetted potential, Phys. Rev. D 97 (2018) 016017 [1710.02319].

[18] X. Du, M. He and R. Rapp, Color screening and regeneration of bottomonia in high-energy heavy-ion collisions, Phys. Rev. C 96 (2017) 054901 [1706. 08670]. 